\title{
A COMPREHENSIVE AND DETAILED ANALYSIS OF TYPES, DESIGN AND FUNCTIONALITY OF PARTICLE ACCELERATORS ${ }^{1}$
}

\section{HARDIK CHAUDHARY}

DOI: $10.37648 /$ ijrst.v10i01.004

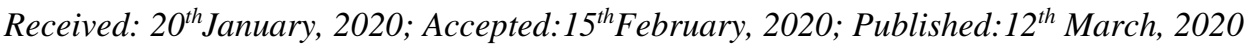

\begin{abstract}
A Particle Accelerator is a device which uses electromagnetic fields to propel charged particles or ions to high speeds and contain them in well-defined paths in the form of beams. These particle accelerators also provide us with a new developed field of electronics called the 'Spintronics'. Unlike conventional devices that use electrons' charge to create power, Spintronic devices use electrons' spin. Thus these accelerators provide wide range of possibilities of applications in industrial and particularly in electronics. Its application also lies in generation of high ranges of radio frequencies especially for microwave electronic devices.
\end{abstract}

\section{INTRODUCTION}

During the $21^{\text {st }}$ century, the infinite need for higher-energy beams for basic research in various fields has been a driving force in the advancement of particle accelerators' technology. The term particle accelerators or particle colliders in modern physics are used to refer to devices that speed up small atomic particles to the velocity of light and maintain them in form of small consistent beams by electromagnetic waves, which pushes the particles in a forward direction (Cousineau, Holme \& Zhang, 2016). The outcome of particle acceleration is that kinetic energy in the subatomic particles after the collision is converted to different forms of nuclear radiation. The first particle accelerator device was developed in the early 1920s and comprised of two electrodes placed inside a vacuum vessel with a potential drop of $100 \mathrm{kV}$ (Wilson, 2013). There are two primary types of these devices namely linear and circular type. This piece of work seeks to analyse the physics of particle accelerators; types, design, and functionality to prove that researcher's intellectual inquisitiveness for basic physics laws has garnered technical advances on accelerators applications in various fields of science.

\footnotetext{
${ }^{1}$ How to cite the article: Chaudhary H., A Comprehensive and Detailed Analysis of Types, Design and Functionality of Particle Accelerators, IJRST, Jan-Mar 2020, Vol 10, Issue 1, 28-35, DOI: http://doi.org/10.37648/ijrst.v10i01.004
} 


\section{HOW PARTICLE ACCELERATORS WORK}

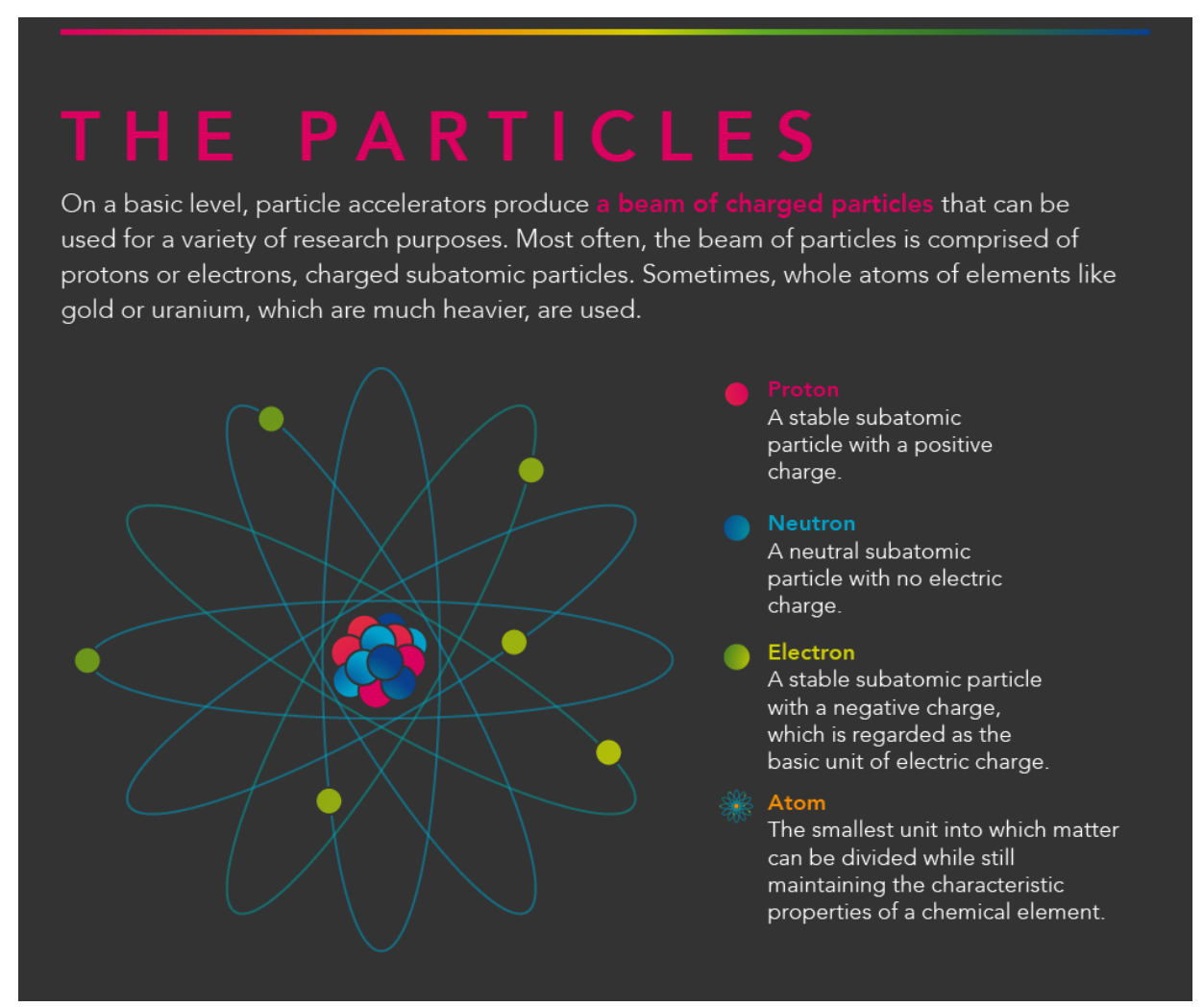

Even though there are different types of particle accelerators, they all have the same operational and functional mechanism outlined below. The intended use of an accelerator dictates the choice of the subatomic particles and the desired beam properties. The particles to be selected are always electrically charged electrons, positrons, nuclei, ions or protons (Wilson, 2013). However, on a basic level, accelerators generate mainly generates a beam made of electrons and protons ("How particle accelerators work", 2014). A proton can be defined as a stable constituent of an atom with a positive charge, whereas an electron may be described as a negatively charged particle and may be considered as the basic unit of an electric charge. The fundamental parts of an accelerator include a source (1), magnets (3), vacuum chambers (2), targets (5), detectors (6), electric fields (4), cooling systems, injection and extraction systems, beam diagnostics and accelerating structures as shown in figure 1 .

The source produce charged particles that ought to be accelerated and direct them in vacuum chambers. The vacuum component is a highly evacuated pipe with minimum pressure to reduce scattering of the particles. Powerful magnets along the track are used to direct the beam particles along the desired route by bending and absorbing them in a narrow beam. The absence of electric field outside the vacuum chamber creates a potential difference that enables the accelerating structures to speed up the beam particles. 


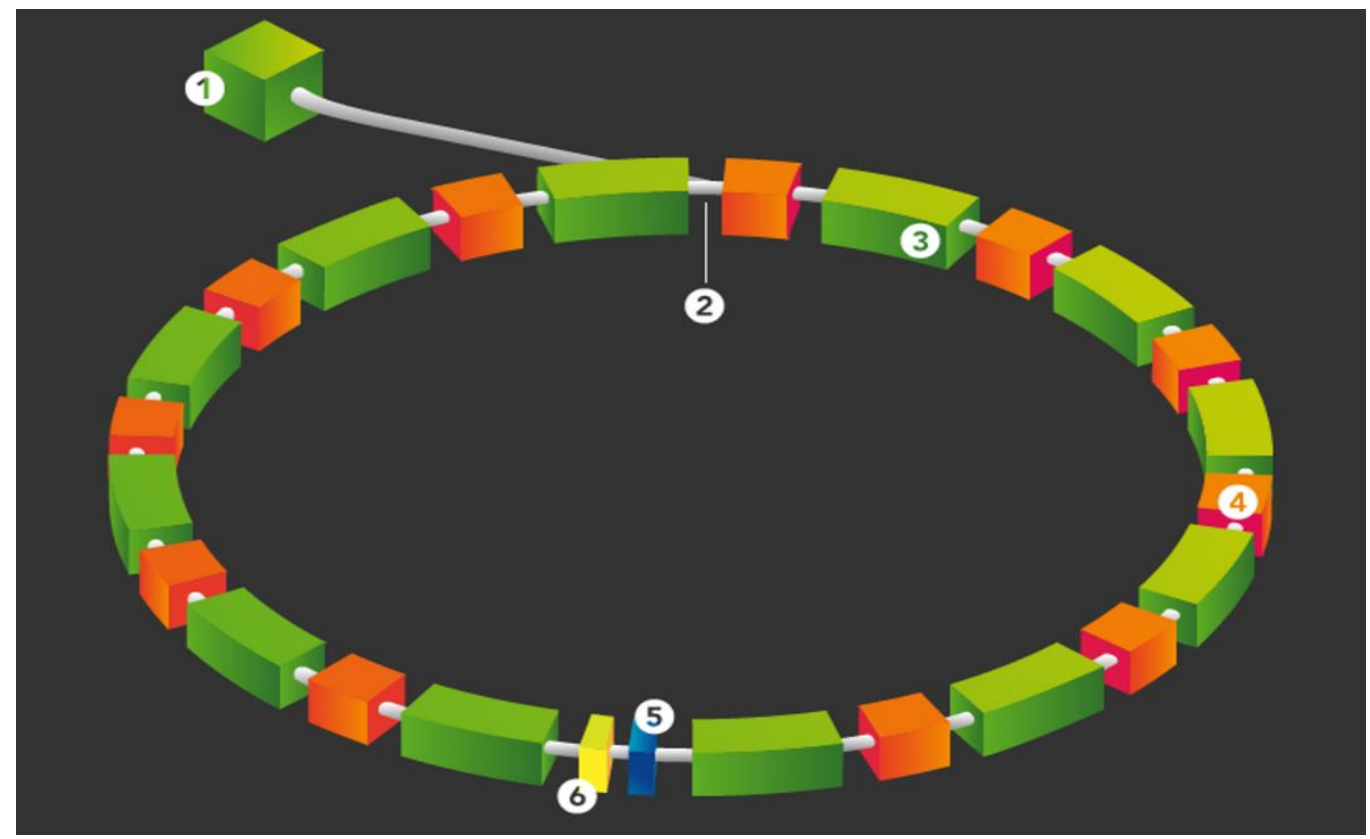

Figure 1. Basic parts of a particle accelerator

The potential difference ( $\mathrm{pd}$ ) formed along the tubes of an accelerator can be varied from negative to positive at under certain frequencies to form electromagnetic waves that can be accelerated in form of bunches (Dotson, 2014). After attaining the desired speeds, particles are either directed at a fixed target or collide two particle beams (Dotson, 2014). In most settings, the fixed target is usually a thin piece of metal foil (Dotson, 2014).

Heat dissipated by the accelerator is removed by ultra-low temperature helium to ascertain that superconductors magnets and accelerating structures achieve the desired superconductivity. Injector and ejection systems are used to guide particles in or out of one accelerator to another (Wiedemann, 2015). Beam diagnostics serves an integral function of providing information about the intensity, position, loss, and profile of the beam ("Accelerators and Beams", n.d.).Beam intensity in the accelerator is expressed in terms of electrical current as a ratio of charge passing a current monitor per time. Over a complete cycle of an accelerator, the beam current is averaged to give average beam current.

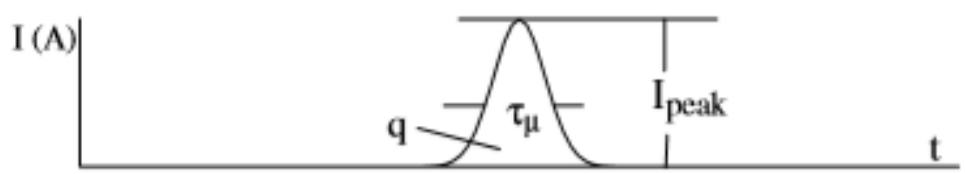

Figure 1a. Peak current

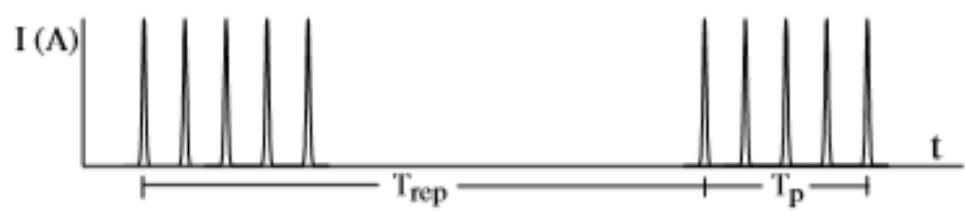

Figure 1b. Average current 
During emission of particles, the radiation causes the reaction force on the emitting surface to reduce gradually due to loss of energy via emission of many photons. The emission of photons in a transverse plane causes loss of longitudinal momentum resulting in a phenomenon known as damping (Wiedemann, 2015). For a particle with initial momentum po and a traverse momentum $\mathrm{p} 1$, the loss of momentum leads to $-\Delta \mathrm{p}$ as shown below in figure 2 . The energy lost is compensated by the acceleration of the particle.

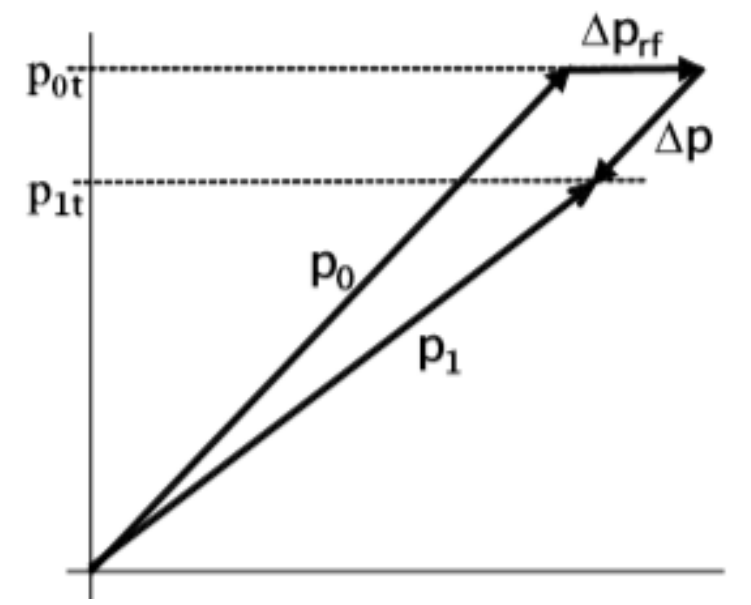

Figure 2. Reduction of momentum and energy loss

The divergence, width, length or energy spread are beam properties that vary with emittance, rf-parameter, lattice and the type of accelerator used. A beam particle at any point can be represented using few phase ellipses for different momentum due to energy loss as shown in figure 3 (Wiedemann, 2015). The phase ellipses for various particle momentum may be shifted proportionally to the dispersion function at a given point.

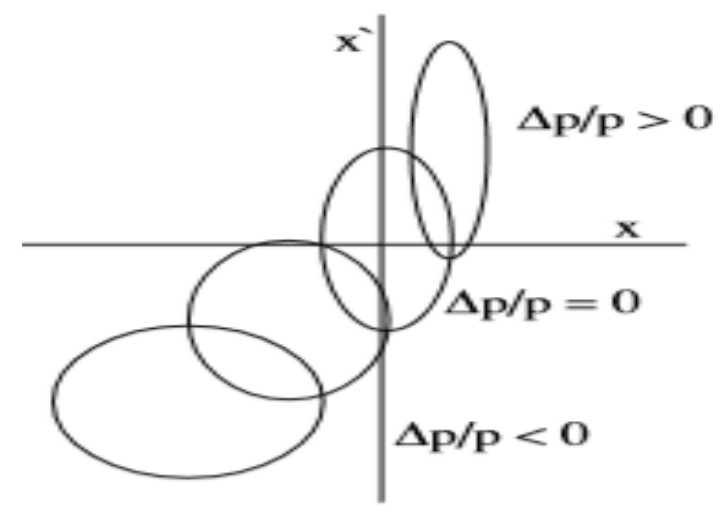

Figure 3. Beam ellipses for various emittance

The unification of particles in accelerators may be achieved by using particles with a mass greater than $100 \mathrm{GeV}$. Figure 4 below shows how diverged couplings look like when particles of mass greater than $100 \mathrm{GeV}$ are used. 


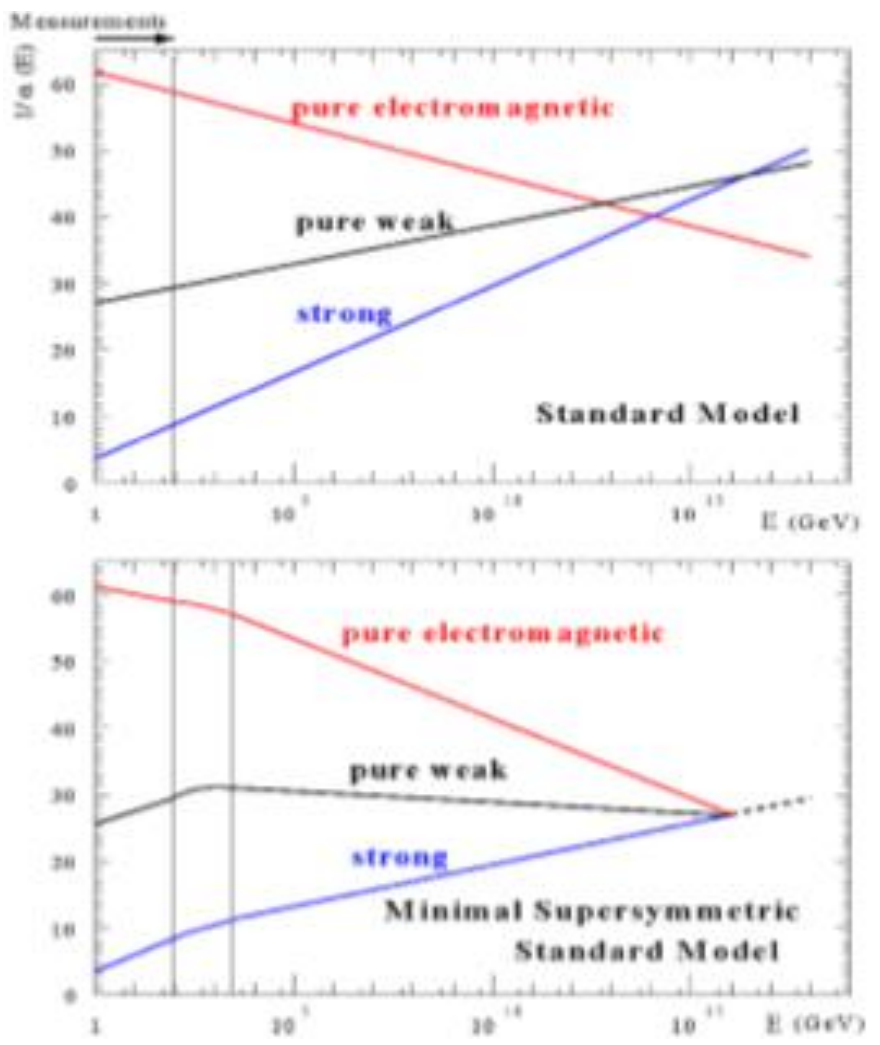

Figure 4. Unification of particles using particles with mass greater than $100 \mathrm{GeV}$

\section{TYPES OF PARTICLE ACCELERATORS}

\section{Radio Frequency Linear Type Accelerators}

The Radio Frequency (RF) Linear type particle accelerators are also known as linac and employ microwave technology to speed up electrons in the waveguide, which is a major component of the device.

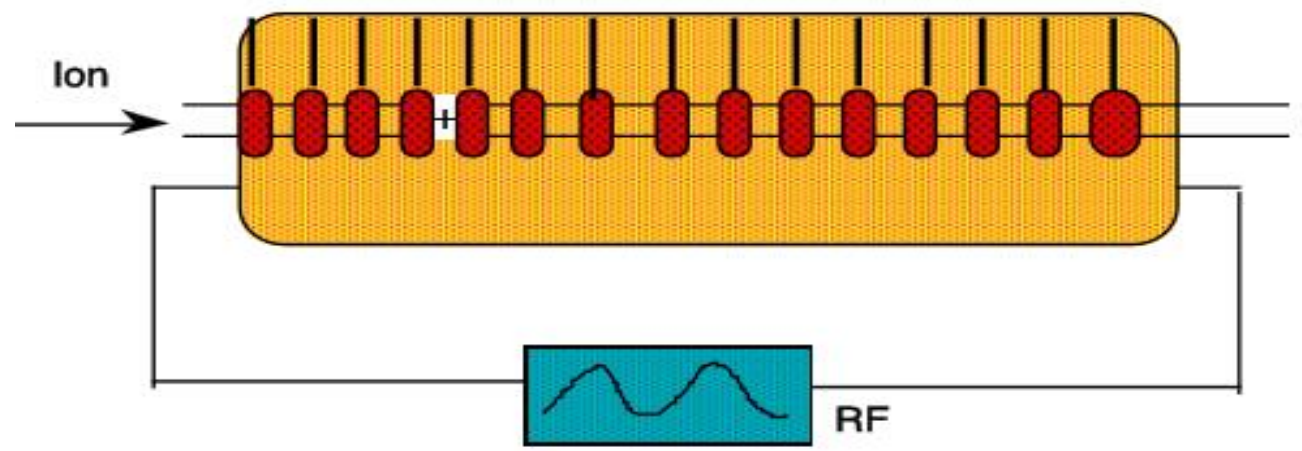

Figure 5. An accelerating portion of a linear accelerator

Subatomic particles move along a straight vacuum track or copper tubes and eventually collide with the target. The hollow tubes are enclosed and evacuated to prevent the formation of electric field guaranteeing that the charged atoms move with uniform velocity through the wave-guide. An alternating current voltage is applied to the tubes so that potential difference of varying magnitude and direction is formed.

The velocity of small charged particles in different tubes is increased uniformly until they travel with voltage waves along the linac. When the beam entrapped within the tube reaches the end of the tube, detectors placed at 
predetermined points are used to record the type of radiation is released. One of its types is the Stanford University linac, $3200 \mathrm{~m}$ long and with an ability to accelerate charged electrons to an energy of up to $50 \mathrm{GeV}$. The design of Stanford's linac permits collision of two beams of particles which are accelerated and momentarily kept in the storage rings. It is noteworthy that the linac can only be used for fixed-target experiments.

\section{Disadvantages}

The traverse mode impendence of the cells and the flatness of the voltage cell are the predominant problems in the design of linear particle accelerators. Additionally, linear accelerators devices are long and necessitate large storage facilities. The linac type needs to be powered often, therefore a stable power supply not only increases the initial installation costs but also the maintenance labor. When the walls of the tubes are made of a good conductor, there is a likelihood of formation of large electric fields around the accelerator. When this occurs, the resistance of the walls rapidly changes the electrical energy to heat energy which should be cooled using coolants. Nevertheless, superconductors tend to be expensive, especially for large device thus a balance between the two must be established to ensure that there is no electric field along the track.

\section{Circular Type Accelerators}

A circular particle accelerator is another basic type where atomic particles move along a circular track until they hit the target. Particles are propelled to travel along the circle several times such that a magnetic field across the track is increased after each pass to ensure that the confined beam is accelerated. Once the desired power level has been achieved, detectors are put along the circular track to record data. The cyclotron can be used either in fixed target or colliding beams settings.

\section{Model Example}

Lawrence's Cyclotron is a typical example of circular particle accelerators. Even though a cyclotron is somewhat like a linear particle accelerator wrapped into a spiral, it does not have many chambers but instead has only two vacuum chambers referred to as dees. The two chambers assume a capital letter D's shape back to back. Subatomic particles in the cyclotron are repeatedly propelled via circular tubes the magnitude of electric field increasing after each pass, thus increases the acceleration of the beam (Dotson, 2014). Once the particles attain the required energy levels, a target is placed in predetermined positions along the tubes and a detector used to observe the nature of the collision.

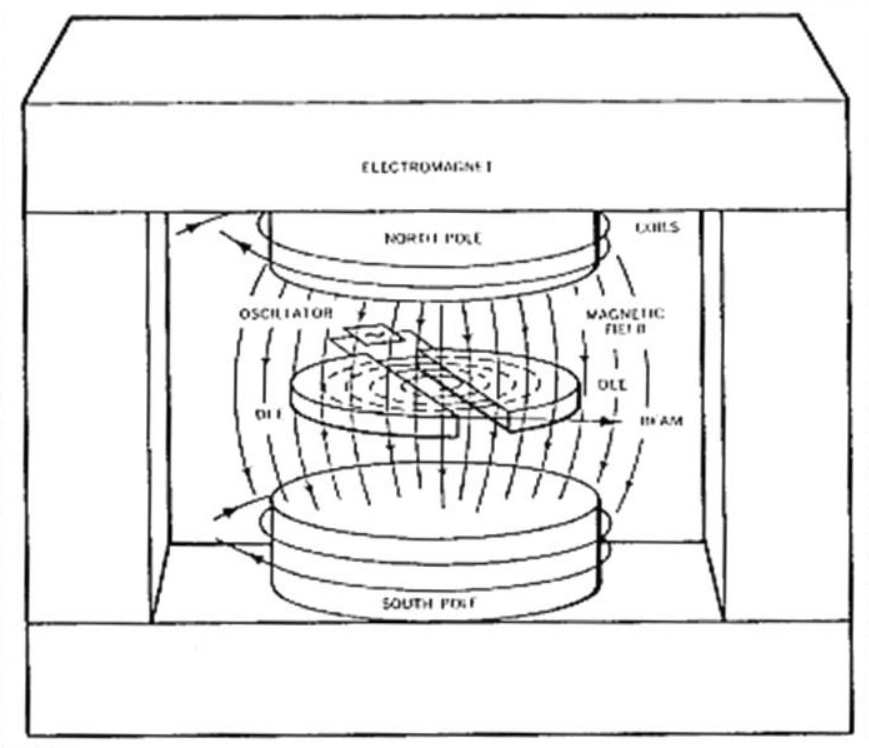

Figure 6. A schematic of a Cyclotron 
The device has powerful electromagnets that produce a strong magnetic field, which keeps the particles revolving around the circular track. The cyclotron is designed such that the potential difference between the dees continually alternates in direction. The constant change in direction ensures that at every time when the charged atoms reach the gap, they experience a forward acceleration thus increasing the beam's velocity. Within the half of each revolution, the particles in each dee move with uniform speed to ascertain that the particle gain energy. Once the beam has gained energy, it spirals out towards the edge of the accelerator and then exits. The K1200 is an example of a powerful modern cyclotron with an ability to accelerate the nuclei to an energy of approximately $8 \mathrm{GeV}$.

\section{Advantages of Cyclotron over the Linac}

The main advantage of cyclotrons is that they have one electrical driver. This implies that power, construction, and maintenance costs are saved and may be allocated to improve the efficiency of the device. Compared with the linac, cyclotrons tend to produce a constant stream of subatomic pulses at the target thus their average power is moderately high. The design of cyclotrons entails a compact device compared to the linac thus lowering the installation, maintenance and shielding expenses.

\section{APPLICATIONS OF PARTICLE ACCELERATORS}

Accelerators have proved to have had an integral role in particle and nuclear physics discoveries. Beams resulting from particle accelerators have a wide range of applications in industrial and medical research. Thousands of cancer patients across the world are treated using beams obtained from particle accelerators (Dotson, 2014). In addition to this, it is known that accelerators generate short-lived radioactive elements known as isotopes, which are used in millions of diagnostic medical procedures and laboratory tests ("Accelerators and Beams", n.d.). Advancement in proton-ion beam therapy technology in recent years has enabled doctors to cure cancer cells without harming vital tissues in the body. Dental industry depends on accelerators to produce Xrays used in dental screening. It is thus rational to contend that nuclear diagnosis and radiotherapy in the field of medicine not only generates a considerable amount of revenue but also saves millions of lives.

Public health and food industry have used the technology behind particle accelerators to irradiate food to prevent deaths resulting from food-borne diseases. Electron beams are used to sterilize products before packaging to prevent bacterial contamination. In the semiconductor industry, ion beams developed using particle accelerators are used to add special atoms semi-conductors such as in the production of hard surface layers in artificial hip and knee joints, cutting tools and high-speed bearings (Dotson, 2014).

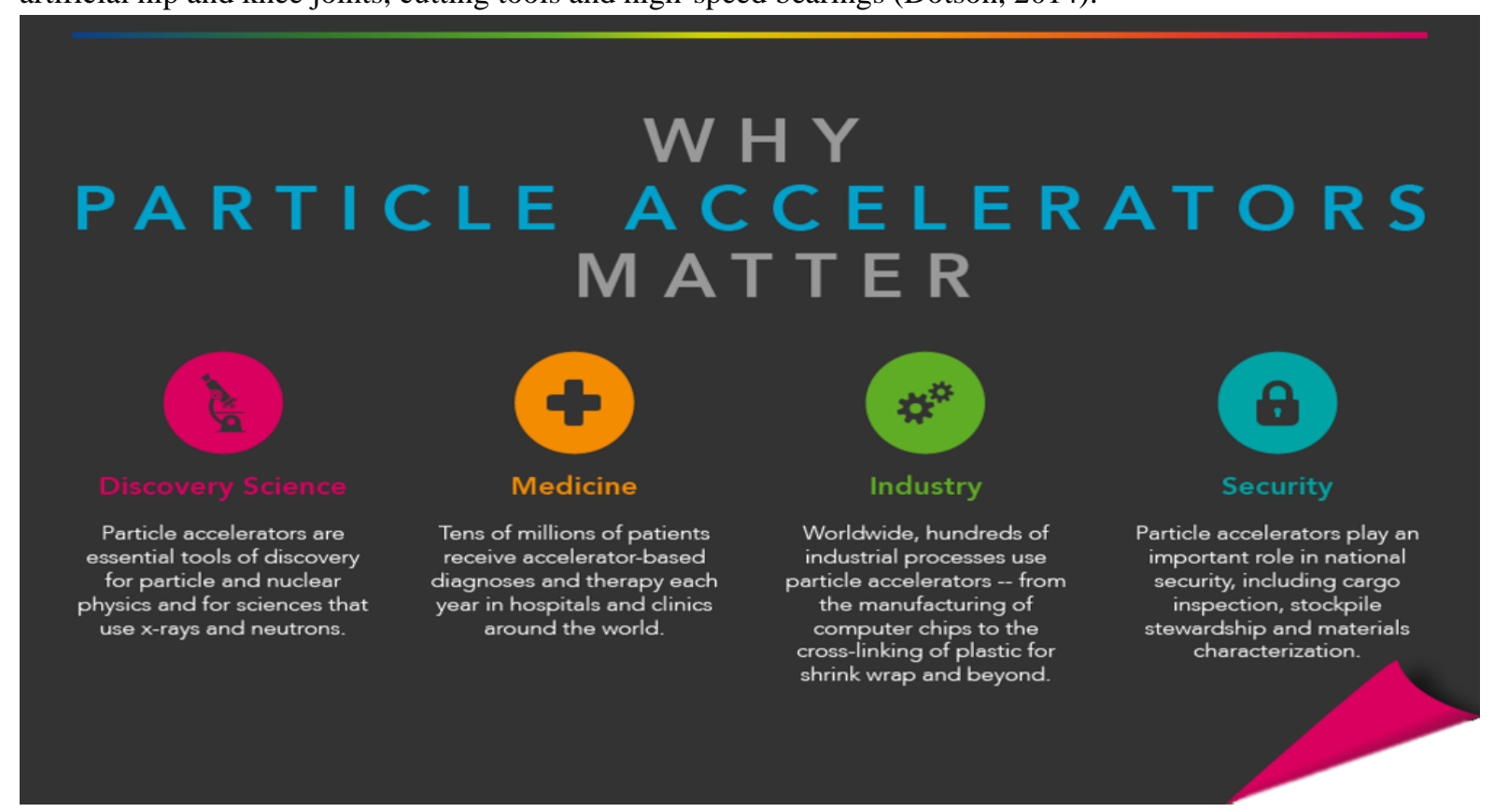


Thirdly, accelerators have found a promising spot in archaeology and pharmaceutical research since they provide precise fossil, DNA and sample data in a non-destructive way. In high energy physics, accelerators have produced answers to questions regarding the universe, physical laws that tend to control space, time, matter and energy (Dotson, 2014). Scientists have discovered that accelerators may be used to produce photons; atomic particles responsible for magnetic radiation. This idea has been used to cut across many energy fields and apparently, particles accelerators are probable avenues towards cracking energy snags that have lasted for centuries.

\section{CONCLUSION}

Most of what people in the field of physics know and learn about the basic nature of matter is learned by probing it with directed beams. Particle accelerators have changed the view of nature and the world we live in and have become a fundamental part of any nation's technical infrastructure ("Accelerators and Beams", n.d.). The basic types of accelerators widely used in modern technology are the Cyclotron and the Radio Frequency Linear Accelerators. They are integral tools of modern research and science and have found a diverse range of applications in human life: medicine, food technology, pharmaceuticals, and archaeology. Thus, particle accelerators are and will remain pertinent in human life as they are pivotal in understanding the cosmos and providing information required to falsify new theories.

\section{REFERENCES}

[1] Accelerators and Beams. (2010). Retrieved from https://www.jlab.org/sites/default/files/APS.pdf

[2] Dotson, B. (2014). How Particle Accelerators Work. Energy. Retrieved from https://www.energy.gov/articles/how-particle-accelerators-work

[3] Wilson, L. (2014). Accelerators and Beams: Tools of Discovery and Innovation. Washington, DC: Division of Physics of Beams of the American Physical Society.

[4] Wiedemann, H. (2015). Particle accelerator physics. New York: Springer Stanford University.

[5] Cousineau, S., Holme, J., \& Zhang, Y. (2016). Introduction to Accelerators: Evolution of accelerators and modern day application. Journal of Aetherometric Research, 2(3), 12-34. 